\title{
Optimizing Translation of Health Research into Practice: A systematic review of reviews
}

\author{
Hammoda Abu-Odah ${ }^{1}$, Nizar Said ${ }^{2}$, Satish Chandrasekhar Nair ${ }^{3}$, Matthew J Allsop ${ }^{4}$, \\ David Currow ${ }^{5}$, Motasem Said Salah ${ }^{6}$, Bassam Abu Hamad ${ }^{7}$, Khamis Elessi ${ }^{8}$, Ali \\ Alkhatib $^{9}$, and Yousuf ElMokhallalati ${ }^{4}$ \\ ${ }^{1}$ The Hong Kong Polytechnic University \\ ${ }^{2}$ Al-Najah National University \\ ${ }^{3}$ Tawam Hospital \\ ${ }^{4}$ University of Leeds \\ ${ }^{5}$ University of Technology Sydney \\ ${ }^{6}$ Palestinian Ministry of Health \\ ${ }^{7} \mathrm{Al}$ Quds University \\ ${ }^{8}$ Islamic University of Gaza \\ ${ }^{9}$ University College of Applied Sciences
}

July 30, 2020

\begin{abstract}
Abstract Rationale, Aims, and Objectives Effective translation of knowledge that is generated from health research into clinical practice has the potential to enhance the quality of care, health services delivery, and its cost-effectiveness. Critical barriers limiting translation of health research into practice are embedded both at the individual and the organizational level. An understanding of the embedded facilitators and barriers will enable findings from health research to be implemented into practice in a timely way. This systematic review aims to synthesize evidence from existing reviews about barriers and facilitators that influence the uptake of health research findings into clinical practice. Method A systematic review was conducted by following PRISMA guidelines. The PubMed, EMBASE, SCOPUS, Web of Sciences, and CINAHL databases were searched to identify review papers published from inception to February 2020. The innovative care for chronic conditions was the analytical framework for data analysis. Results The search yielded ten publications. Translation of new evidence was limited predominantly by individual-related issues and less frequently organizational factors. Inadequate knowledge and skills of individuals to conduct, organize, utilize, and appraise research literature, and lack of resources were the primary individual and organizational challenges. This review also discloses mistrust by policymakers about the potential of research to translate into practice, affecting both the development of health policies and also systematic public investments for research programs. A sizable proportion of mistrust by the policymakers stems from their lack of knowledge for understanding research methods and limited skills in comparing research outcomes. To circumvent this barrier, identification and partnering with policymakers and health professionals at all stages of the research process, is critical. Conclusions Despite the challenges affecting the translation of research into practice, utilization of research evidence is critical to improving health services delivery and outcomes. Keywords: policymakers, healthcare professionals, evidence translation
\end{abstract}

\section{Optimizing Translation of Health Research into Practice: A systematic review of reviews}

Hammoda Abu-Odah,,${ }^{1,2^{*}}$ Nizar Said, ${ }^{3}$ Satish Chandrasekhar Nair, ${ }^{4}$ Matthew J Allsop, ${ }^{5}$ David Currow, ${ }^{6}$ Motasem Said Salah, ${ }^{2,7}$ Bassam Abu Hamad, ${ }^{8}$ Khamis Elessi, ${ }^{9}$ Ali Alkhatib, ${ }^{2}$ Yousuf ElMokhallalati, ${ }^{10}$ 
${ }^{1}$ School of Nursing, The Hong Kong Polytechnic University, Hung Hom, Hong Kong

${ }^{2}$ Nursing and Health Sciences Department, University College of Applied Sciences (UCAS), Gaza, Palestine.

${ }^{3}$ Department of Nursing and Midwifery, An Najah National University, Nablus, Palestine

${ }^{4}$ Johns Hopkins Medicine Tawam Hospital, College of Medicine UAE University, Box 15258, Al Ain, United Arab Emirates

${ }^{5}$ Academic Unit of Palliative Care, Leeds Institute of Health Sciences, University of Leeds, Leeds, UK

${ }^{6}$ Improving Palliative, Aged and Chronic Care through Clinical Research and Translation, Faculty of Health, University of Technology Sydney, Ultimo. New South Wales. Australia

${ }^{7}$ Nursing Consultant for Minster of Health, Ministry of Health, Gaza, Palestine

${ }^{8}$ School of Public Health, Al Quds University, Abu Dees, POB 89, Jerusalem, Palestine

${ }^{9}$ Faculty of Medicine, Evidence-Based Medicine Unit, Islamic University, Gaza city, Palestine

${ }^{10}$ Academic Unit of Palliative Care, Leeds Institute of Health Sciences (LIHS), School of Medicine, University of Leeds, Leeds, UK

Hammoda Abu-Odah, Phone: (852) 27664520, Email: hammoda.abuodah@connect.polyu.hk

Nizar Said, Phone: (852) 3400 3792, Email: nizar.said@connect.polyu.hk

Satish C. Nair, Phone: (971) 37074739, Email: satchi2000@outlook.com

Matthew J Allsop, Phone: (+44) 1133434185, Email: m.j.allsop@leeds.ac.uk

David Currow, Phone: (+61) 295145967, Email: david.currow@uts.edu.au

Motasem Said Salah, Phone (972) 594045051, Email: motasem_salah@hotmail.com

Bassam Abu Hamad, Phone (972) 599351515, Email: ghsrcb@gmail.com

Khamis Elessi Phone (972) 599868034, Email: khamis1000000@gmail.com

Ali Alkhatib, Phone (972) 599496492, Email: akhateeb@ucas.edu.ps

Yousuf ElMokhallalati, Phone: (+44) 113343 4996, Email: y.elmokhallalati@leeds.ac.uk

\section{* Corresponding author}

Hammoda Abu-Odah

Hammoda.abuodah@connect.polyu.hk

(852) 27664520

\section{Abstract}

\section{Rationale, Aims, and Objectives}

Effective translation of knowledge that is generated from health research into practice has significant contributions in enhancing health services delivery and its cost-effectiveness. Critical barriers limiting translation of health research into practice are embedded both at the individual and the organisational level. An understanding of the embedded facilitators and barriers will enable findings from health research to be implemented into practice in a timely way. The purpose of this review is to synthesise evidence from literature about the barriers and facilitators on translating health research into practice.

\section{Method}


A systematic review was conducted to identify review studies published from inception to February 2020. Five databases (PubMed, Scopus, Embase, CINAHL and Web of Science) were searched to identify papers. The innovative care for chronic conditions was adopted as an analytical framework.

\section{Results}

The search yielded ten publications. Translation of new evidence was limited predominantly by individualrelated issues and less frequently organisational factors. Inadequate knowledge and skills of individuals to conduct, organise, utilise, and appraise research literature, and lack of resources were the primary individual and organisational challenges. This review also discloses mistrust by policymakers about the potential of research to translate into practice, affecting both the development of health policies and also systematic public investments for research programs. A sizable proportion of mistrust by the policymakers stems from their lack of knowledge for understanding research methods and limited skills in comparing research outcomes. To circumvent this barrier, it is critical for identification and partnering with policymakers and health professionals at all level of the research stages.

\section{Conclusions}

Despite the challenges affecting the translation of knowledge that is generated from health research into practice, the utilisation of research evidence is critical to improving health services delivery and outcomes.

Keywords: policymakers, healthcare professionals, evidence translation

\section{Introduction}

The utilisation of knowledge that is generated from health research to inform and guide clinical practice is recognised as a high global priority. ${ }^{1-3}$ The need for evidence-informed practice is recognised across multiple governments policymakers, and funding agencies in response to a rapidly expanding evidence base, the restructuring of health care, insufficient resources, and increased professional accountability. ${ }^{4}$ Effective translation of knowledge has the potential to improve health outcomes and health services delivery. ${ }^{5-8}$

"Knowledge translation" (KT) is a term utilised to explain the activities involved in translating healthrelated research findings into practice. ${ }^{9,10}$ It is defined as "a dynamic and iterative process that includes the synthesis, dissemination, exchange and ethically sound application of knowledge to improve health, provide more effective health services and products and strengthen the healthcare system". ${ }^{11}$ It attempts to assure that stakeholders or "knowledge users", are familiar with, using and accessing research findings and engaging them as active participants in the research process. ${ }^{12-15}$

Despite the increase in health research globally, the method of translating health-related research findings into policy and practice remains slow, ${ }^{8,16}$ and the gap between findings and practice is growing. ${ }^{17,18}$ These gaps might be related to a wide range of challenges that have been reported in previous reviews. For instance, Oliver and colleagues ${ }^{19}$ explored the challenges of utilising of health research findings by policymakers. They identified several obstacles towards the translation of knowledge into policy. Lavis et $\mathrm{al}^{20}$ reported the significance of involving policymakers with health research activities. Though the two reviews focused on barriers and identified several obstacles in the translation of knowledge, their searches were limited to policymakers' and related factors, without the impact on other stakeholders/users. To the best of our knowledge, there has been no systematic overview to address the challenges of translating health-related research evidence into policy and practice simultaneously.

Therefore, this systematic review aims at synthesising evidence from earlier reports and existing literature about the barriers and facilitators that enable the translation of health research findings into practice. The methodology used is derived from the multi-level WHO Innovative Care for Chronic Conditions (ICCC), ${ }^{21}$ a model widely used across studies with multi-level determinants, such as the micro-, meso- and macro-levels.

\section{Methods}

\section{Study design}


Framework synthesis method ${ }^{22,23}$ was adopted to undertake this systematic review and narrative synthesis. This synthesis method was selected because it offers a robust approach to shaping and synthesising the large amount of textual data produced by research. ${ }^{22,23}$ "Preferred reporting items for systematic reviews and meta-analyses" (PRISMA" guidance (Figure 1) was utilised to structure this review. ${ }^{24}$

\section{Search sources and strategies}

PubMed, Scopus, Embase, CINAHL and Web of Science databases were searched for reviews published in the English from inception to February 2020.

The following group terms were utilised for searching: "translational research" OR "knowledge translation" OR "evidence to practice" OR "knowledge exchange" OR "knowledge interaction" OR "research utilisation" OR "research dissemination" OR "knowledge uptake" OR "knowledge-to-action" OR "research diffusion") AND (medicine OR nursing OR "public health" OR "health") AND (Challenges OR obstacles OR limitations OR problems OR barriers). Expressions for "review*" were also included. A detailed search term for PubMed is displayed in Table 1.

\section{Eligibility criteria}

\section{Inclusion criteria}

- Reviews reporting barriers or facilitators associated with research evidence and clinical practice; and

- Reviews that were written in the English.

\section{Exclusion criteria}

- Non-systematic literature reviews or discussion papers;

- Other types of articles such as protocols, editorial comments, conference abstracts, and policies; and

- Publications where full-text versions could not be obtained.

\section{Study selection and data extraction}

All of papers retrieved from five databases were exported into Endnote version X8. After removing duplications, remaining papers were screened for eligibility criteria by the first and second authors independently. The potentially eligible full- text papers were then located for screening by two authors, and any uncertainty encountered between them was resolve by the third author. Reasons for excluding papers were reported in Figure 1.

The data of included ten papers was extracted by the first author (HAO) into a data extraction sheet developed for this study. The datasheet was used to report the following data; (1) citation information; (2) number of studies included; (3) review aim(s); (4) main findings (challenges and facilitators) summarised based on the ICCC model (Table 2).

\section{Quality appraisal of reviews}

The first two authors (HAO and NS) independently utilised two separate, validated tools for evaluating the methodological quality of the included reviews. The first is "A MeaSurement Tool to Assess systematic Reviews-2" (AMSTAR-2), which was used for assessing systematic and scoping reviews. AMSTAR-2 consists of 16 items; each item ranked into three levels; yes, no or partial yes. The final scores of each included review were rated as "high" which mean that review provides a comprehensive summary of findings, "moderate level mean that review more weakness but not critical flaws, while 'low" level indicates that review has not provided comprehensive findings and has critical flows. ${ }^{25}$ The second tool is the "International Narrative Systematic assessment" (INSA) which was used for assessing narrative review papers. It contains seven items where each item is rated as 'yes' or 'no'. A review with a total score of higher than 5 points is judged as a 'good' quality paper. ${ }^{26}$

\section{Data analysis}


The ICCC was adopted as a framework for the analysis of data. ${ }^{21}$ The framework includes three levels; the first is the micro-level focusing on individual users, the second is meso-level focusing on the healthcare organisation and systems, and finally, the macro-level related to the policies ${ }^{27}$.

\section{Results}

\section{Study characteristics}

A total of 829 articles were identified by searching the five databases. Of these, 790 were excluded because they were ineligible and duplicated. The total number was reduced to 39 articles. Screening of full-text papers excluded an additional 34 articles. A total of five articles were eligible for inclusion the review. Reference list of each included articles was screened to check if any relevant review has been omitted. Five additional articles were included. As a result, ten articles were included for quality appraisal and analysis (Figure 1).

Of the ten included articles, five were systematic reviews, ${ }^{19,28-31}$ four were narrative reviews,, ,32-34, and the one remaining was a scoping review. ${ }^{35}$ Regarding the geographical focus of included reviews, three reviews were focused on developing countries, ${ }^{28,31,33}$ one concentrated on the global perspective, ${ }^{8}$ while the other reviews did not specify the regions. . $^{8,19,29,30,32,34,35}$

Most reviews focused on the policymakers and healthcare system perspectives, ${ }^{19,28,35}$ two reviews were focused on nurses, ${ }^{31,32}$ and, healthcare professionals in general, ${ }^{29,30}$ while one review was focused on clinicians. ${ }^{32}$ With regards to the timing of publication, most reviews were published in the last ten years. ${ }^{8,19,28,30-33,35,36}$ Two reviews were published before $2010 .{ }^{29,34}$

With regards to the number of articles included within each review article, six articles specified the number of studies they included. ${ }^{19,28-31,35}$ Two articles included more than one hundred studies, ${ }^{19,30}$ one review included 62 studies ${ }^{28}$ and the other ${ }^{29,31,35}$ included less than fifty studies; ranged between 16 and 38 studies. The main findings of the included articles are illustrated in Table 2.

\section{Quality appraisal of reviews}

The included systematic reviews ${ }^{19,28-31,35}$ were reported very high quality that suggests reviews showing a robust and accurate summary of the findings. Included narrative reviews ${ }^{8,32-34}$ scored [?] 5 points, reflecting a good quality paper. The quality of each selected article is outlined in Table 2.

\section{Barriers and facilitators}

\section{Micro-level (individuals)}

Personal barriers focused on healthcare professionals abilities to conduct, read, use, or translate evidence into clinical practice. Findings revealed that a lack of professionals engagement in research and lack of time or insufficient critical appraisal skills and an inability of healthcare professionals to utilise the research findings and recommendations into clinical practice were the most common individual-levels challenges. ${ }^{28,31}$ Moreover, the inability of healthcare professionals to use a computer to search and access research databases 32,35 was considered a challenge for professionals seeking to update their knowledge and skills in research and created an obstacle in the translation of research evidence into practice.

Other barriers, such as the unfamiliarity of professionals with evidence-based practice, ${ }^{31}$ lack of interest to update knowledge, ${ }^{35}$ and mistrust of professional of the value of research ${ }^{32,35}$ were further barriers identified at the individual level. Further to the inability of healthcare professionals to interpret findings from studies, there was an inability to understand statistical measures utilised in research. ${ }^{30}$

The facilitators for overcoming the challenges on the mico-level were the motivation of healthcare professionals, ${ }^{29}$ and the interest in study findings, alongside suitable packaging and targeted communication of results. ${ }^{33}$

Meso level (systems or organisations) 
This level includes systems' or organisations' barriers focused on education, training, resources, services, policies, and culture of organisations. Systemic and organisational factors such as time constraints, insufficient organisation resources, poor dissemination, and lack of access to research were the highest common reported barriers. ${ }^{19,28,29,31,32,35}$ Insufficient resources in the form of material and equipment required for the implementation of research and inadequate facilities to conduct research were reported in three studies 28-30,33-35

Inadequate organisational support was the second most frequently cited barrier for translating evidence into practice. Lack of workforce was reported as a challenge to translating evidence into practice. ${ }^{31}$ Insufficient staffing was linked with difficulties of staff to find an appropriate time at work for searching and reading research papers, articles, and guidelines because of heavy workloads. ${ }^{31}$ Other barriers specific to the translation of knowledge to nursing practice were reported, including a lack of research training courses, ${ }^{31}$ and dearth of interaction among educational and clinical environments. ${ }^{31}$

Institutional support for the translation of evidence into practice can come through development capacitybuilding workshops, ${ }^{28}$ dissemination of main research findings across organisations, ${ }^{19,33}$ and budgeting for research activities such as face-to-face meetings. Another important facilitator was the development of a pathway of communication to build a shared understanding of the work. ${ }^{35}$

\section{Macro-level (economic and political)}

This level focused on policymakers' issues that support translating evidence into practice such as guidelines, partnerships and regulations. Three reviews reported political challenges as factors impeding translation of health research evidence into clinical practice. ${ }^{19,34,35}$ Policymakers were not trained and skilled in research methods. ${ }^{19}$ Policymakers did not see alignment between research and policy considerations, ${ }^{35}$ and remain suspicious about the utility of research findings. ${ }^{34}$

Identifying the right stakeholders and developing strong collaboration and connections between policymakers and research staff were the main facilitators discussed in two reviews, ${ }^{19,35}$ These can be achieved through technology support, such as web-based conferencing platforms. This technology provides policymakers with updated information in research and engages them in all research priorities, which help them to make evidence-based choices. ${ }^{35}$ Other facilitators play a significant role in translating evidence into practice, such as developing trust across policymakers and researchers, ${ }^{35}$ developing guidelines that promote best practice. ${ }^{34}$ Important too is early involvement of stakeholders in the design of research, including involvement from the point of research initiation of those most likely to be affected by research output. ${ }^{33}$

\section{Discussion}

This is the first systematic review of reviews to provide a comprehensive and systematic mapping of the challenges related to translating health research and evidence into clinical practice, considered alongside barriers relating to translation into health policy. This review goes further to articulate possible facilitators that enable the translation of health research evidence into practice as expressed across included studies.

Despite convincing evidence from health research, translating evidence into clinical practice or policy can encounter multiple barriers. Translation to the real-world environments is critical for the success of any clinical practice or implementation of health policy. Research settings may adopt a design to limit the influence of uncontrolled variables, thereby limiting real-world influences on research. Evidence-based research alluded to the reduction in cardiac event mortality following the timely use of clopidogrel, yet, the response time was critical for mortality prevention in the context of real-world settings. ${ }^{37}$ Despite the existence of research evidence, clinicians have not been ready to perceive the full potential of statins. This may be attributed to the low adherence rates by patients, compared to those stated in the research evidence. This reflects the fact that research protocols do not reflect real life. Many factors may play a significant role in adherence levels such as motivation and access to medication access. ${ }^{37}$

Ten reviews highlighted that translating health research evidence into clinical practice is affected by several challenges, predominantly contributed by individual-related issues, followed by organisational factors. Ex- 
isting barriers are further compounded by various professionals' overall inadequacy of knowledge and skills to conduct, organise, utilise, and appraise research literature, vital to achieving the translation of health research evidence into clinical practice. Lack of education leading to disinterest, ${ }^{88,39}$ motivational challenges and suspicion over the potential of research evidence to be translated into clinical practice are additional professionals barriers reported. ${ }^{39,40}$ Translational barriers may be reduced by motivating healthcare professionals (micro-level). Individual-level facilitators involve a clear understanding of the target population, who could benefit from the research findings, so that the research evidence can be customised and communicated in an effective module, to enable easy translation. Our results are in accordance with the previous reports indicating successful dissemination and utilisation of research evidence following the identification of the appropriate audience and tailoring messages using appropriate mediums. ${ }^{41-43}$

At an organisational-level, translating evidence-based research into practice requires enormous resources and adequate time. Lack of resources such as availability of research databases and publications are organisationlevel barriers for the translation of research into practice, also indirectly affecting professional skills. Time constraints and workload pressure, and lack of adequate workforce to read and understand research processes limit the translation of research into practice. Our findings have also been supported by other published reports. ${ }^{28,40}$

Our observations indicate mistrust by policymakers about the potential of research to translate into practice. This affects both the development of health policies and also systematic public investments for research programs. A sizable proportion of mistrust by the policymakers stems from their lack of knowledge for understanding research methods and limited skills in comparing research outcomes. Early identification and partnering with all the stakeholders (policymakers and beneficiaries of research such as the community) may overcome this challenge. Similar models have been suggested in earlier studies. ${ }^{44,45}$ Technology-driven interactive models provide all the stakeholders and beneficiaries with constant engagement and updating of information, to enable them to support evidence-based models. ${ }^{42,46-49}$

\section{Strengths and limitations}

Adopted a deductive approach to coding the findings across the research so may not have allowed for new and emergent insights to arise from the literature, but the framework was a useful way of structuring and mapping the findings in this area. The strength of our study is also the identification of the barriers and facilitators at three levels. Despite the challenges affecting the translation of research into practice, our observation and others support ${ }^{50}$ the fact that research evidence is worthwhile for the desired improvements in health outcomes. Although, there can be no one model to organise the translation of research into clinical practice, limiting the influence of the confounding variables (barriers) can effectively cross the chasm of uncertainties.

\section{Conclusion}

This review summarises the key barriers and facilitators for the translation of research to practice both at the individual and organisational levels. Despite the barriers limiting the translation of research into practice, our observation and others support the fact that research evidence is worthwhile for the desired improvements in health outcomes. Limiting the barriers through effective collaboration and cooperation between all stakeholders should aid better translation of health research findings into clinical practice.

\section{References}

1. Conalogue DM, Kinn S, Mulligan J-A, McNeil M. International consultation on long-term global health research priorities, research capacity and research uptake in developing countries. Health Res Policy Syst. 2017;15(1):24-24.

2. Poot CC, van der Kleij RM, Brakema EA, et al. From research to evidence-informed decision making: a systematic approach. Journal of Public Health. 2018;40(suppl_1):i3-i12.

3. Brownson RC, Fielding JE, Green LW. Building capacity for evidence-based public health: reconciling 
the pulls of practice and the push of research. Annual review of public health. 2018;39:27-53.

4. Milner M, Estabrooks CA, Myrick F. Research utilisation and clinical nurse educators: a systematic review. Journal of Evaluation in Clinical Practice. 2006;12(6):639-655.

5. El-Jardali F, Lavis J, Moat K, Pantoja T, Ataya N. Capturing lessons learned from evidence-to-policy initiatives through structured reflection. Health Res Policy Syst. 2014;12:2-2.

6. Langlois EV, Becerril Montekio V, Young T, Song K, Alcalde-Rabanal J, Tran N. Enhancing evidence informed policymaking in complex health systems: lessons from multi-site collaborative approaches. Health Res Policy Syst. 2016;14:20-20.

7. Barratt H, Shaw J, Simpson L, Bhatia S, Fulop N. Health services research: building capacity to meet the needs of the health care system.J Health Serv Res Policy. 2017;22(4):243-249.

8. Straus SE, Tetroe JM, Graham ID. Knowledge translation is the use of knowledge in health care decision making. Journal of Clinical Epidemiology. 2011;64(1):6-10.

9. McKibbon KA, Lokker C, Wilczynski NL, et al. A cross-sectional study of the number and frequency of terms used to refer to knowledge translation in a body of health literature in 2006: a Tower of Babel?.Implement Sci. 2010;5:16-16.

10. Gervais M-J, Marion C, Dagenais C, Chiocchio F, Houlfort N. Dealing with the complexity of evaluating knowledge transfer strategies: Guiding principles for developing valid instruments. Research Evaluation.2015;25(1):62-69.

11. The Canadian Institutes of Health Research. 2016; https://cihr-irsc.gc.ca/e/29418.html. Accessed 22. February, 2020.

12. Greenhalgh T, Wieringa S. Is it time to drop the 'knowledge translation' metaphor? A critical literature review. J R Soc Med.2011;104(12):501-509.

13. Kothari A, Wathen CN. A critical second look at integrated knowledge translation. Health Policy. 2013;109(2):187-191.

14. Engebretsen E, Sandset TJ, Ødemark J. Expanding the knowledge translation metaphor. Health Res Policy Syst. 2017;15(1):19-19.

15. Kreindler SA. Advancing the evaluation of integrated knowledge translation. Health Res Policy Syst. 2018;16(1):104-104.

16. Grimshaw JM, Eccles MP, Lavis JN, Hill SJ, Squires JE. Knowledge translation of research findings. Implement Sci. 2012;7:50-50.

17. Grol R, Grimshaw J. From best evidence to best practice: effective implementation of change in patients' care. Lancet.2003;362(9391):1225-1230.

18. Green LA, Seifert CM. Translation of research into practice: why we can't "just do it". J Am Board Fam Pract. 2005;18(6):541-545.

19. Oliver K, Innvar S, Lorenc T, Woodman J, Thomas J. A systematic review of barriers to and facilitators of the use of evidence by policymakers. BMC health services research. 2014;14(1):2.

20. Lavis JN, Oxman AD, Lewin S, Fretheim A. SUPPORT Tools for evidence-informed health Policymaking (STP) 3: Setting priorities for supporting evidence-informed policymaking. Health Res Policy Syst. 2009;7 Suppl 1(Suppl 1):S3-S3.

21. World Health Organization. Innovative care for chronic conditions: Building blocks for actions: global report. 2002. Google Scholar.2016. 
22. Pope C, Ziebland S, Mays N. Qualitative research in health care. Analysing qualitative data. BMJ. 2000;320(7227):114-116.

23. Barnett-Page E, Thomas J. Methods for the synthesis of qualitative research: a critical review. $B M C$ Medical Research Methodology.2009;9(1):59.

24. Moher D, Liberati A, Tetzlaff J, Altman DG. Preferred reporting items for systematic reviews and meta-analyses: the PRISMA statement.PLoS medicine. 2009;6(7):e1000097.

25. Shea BJ, Reeves BC, Wells G, et al. AMSTAR 2: a critical appraisal tool for systematic reviews that include randomised or non-randomised studies of healthcare interventions, or both. BMJ (Clinical research ed). 2017;358:j4008.

26. La Torre G, Backhaus I, Mannocci A. Rating for narrative reviews: concept and development of the International Narrative Systematic Assessment tool. La Torre G, Backhaus I, Mannocci A/Senses Sc.2015;2(2):3135.

27. Golden SD, Earp JAL. Social ecological approaches to individuals and their contexts: twenty years of health education \& behavior health promotion interventions. Health Education 83 Behavior.2012;39(3):364372.

28. Edwards A, Zweigenthal V, Olivier J. Evidence map of knowledge translation strategies, outcomes, facilitators and barriers in African health systems. Health Res Policy Syst. 2019;17(1).

29. Légaré F, Ratté S, Gravel K, Graham ID. Barriers and facilitators to implementing shared decisionmaking in clinical practice: Update of a systematic review of health professionals' perceptions. Patient education and counseling. 2008;73(3):526-535.

30. Sadeghi-Bazargani H, Tabrizi JS, Azami-Aghdash S. Barriers to evidence-based medicine: a systematic review. Journal of Evaluation in Clinical Practice. 2014;20(6):793-802.

31. Shayan SJ, Kiwanuka F, Nakaye Z. Barriers associated with evidence-based practice among nurses in lowand middle-income countries: a systematic review. Worldviews on Evidence-Based Nursing. 2019;16(1):1220.

32. Athanasakis E. Nurses' research behavior and barriers to research utilisation into clinical nursing practice: a closer look.International Journal of Caring Sciences. 2013;6(1):16-28.

33. Derman RJ, Jaeger FJ. Overcoming challenges to dissemination and implementation of research findings in under-resourced countries.Reproductive Health. 2018;15(1):86.

34. Kalassian KG, Dremsizov T, Angus DC. Translating research evidence into clinical practice: new challenges for critical care. Crit Care. 2002;6(1):11-14.

35. Lawrence LM, Bishop A, Curran J. Integrated Knowledge Translation with Public Health Policy Makers: A Scoping Review. Healthcare Policy. 2019;14(3):55-77.

36. Woolf SH, Purnell JQ, Simon SM, et al. Translating evidence into population health improvement: strategies and barriers. Annual review of public health. 2015;36:463-482.

37. Ma TK, Lam YY, Tan VP, Yan BP. Variability in response to clopidogrel: how important are pharmacogenetics and drug interactions? British journal of clinical pharmacology. 2011;72(4):697-706.

38. Wallis L. Barriers to implementing evidence-based practice remain high for U.S. nurses: getting past "we've always done it this way" is crucial. The American journal of nursing. 2012;112(12):15.

39. Curtis K, Fry M, Shaban RZ, Considine J. Translating research findings to clinical nursing practice. Journal of clinical nursing. 2017;26(5-6):862-872. 
40. Bahadori M, Raadabadi M, Ravangard R, Mahaki B. The barriers to the application of the research findings from the nurses' perspective: A case study in a teaching hospital. Journal of education and health promotion. 2016;5:14-14.

41. Curtis K, Fry M, Shaban RZ, Considine J. Translating research findings to clinical nursing practice. 2017;26(5-6):862-872.

42. Van der Graaf P, Francis O, Doe E, Barrett E, O'Rorke M, Docherty G. Structural approaches to knowledge exchange: comparing practices across five centres of excellence in public health. Journal of Public Health. 2018;40(suppl_1):i31-i38.

43. Kothari A, Wathen CN. Integrated knowledge translation: digging deeper, moving forward. Journal of epidemiology and community health. 2017;71(6):619-623.

44. Ongolo-Zogo P, Lavis JN, Tomson G, Sewankambo NK. Assessing the influence of knowledge translation platforms on health system policy processes to achieve the health millennium development goals in Cameroon and Uganda: a comparative case study. Health policy and planning.2018;33(4):539-554.

45. Oxman AD, Vandvik PO, Lavis JN, Fretheim A, Lewin S. SUPPORT Tools for evidence-informed health Policymaking (STP) 2: Improving how your organisation supports the use of research evidence to inform policymaking. Health Res Policy Syst. 2009;7 Suppl 1(Suppl 1):S2.

46. Dobbins M, Robeson P, Ciliska D, et al. A description of a knowledge broker role implemented as part of a randomised controlled trial evaluating three knowledge translation strategies. Implement Sci.2009;4:23.

47. Haynes AS, Derrick GE, Redman S, et al. Identifying trustworthy experts: how do policymakers find and assess public health researchers worth consulting or collaborating with? PLoS One.2012;7(3):e32665.

48. West RM, House AO, Keen J, Ward VL. Using the structure of social networks to map inter-agency relationships in public health services.Social science \&5 medicine (1982). 2015;145:107-114.

49. Cairney P, Oliver K. Evidence-based policymaking is not like evidence-based medicine, so how far should you go to bridge the divide between evidence and policy? Health Res Policy Syst.2017;15(1):35.

50. Farquhar CM, Stryer D, Slutsky J. Translating research into practice: the future ahead. International Journal for Quality in Health Care. 2002;14(3):233-249.

\section{Declaration of conflicting interests}

The Author(s) declare(s) that there is no conflict of interest'.

\section{Ethics approval}

Not applicable.

\section{Funding}

This research received no specific grant from any funding agency in the public, commercial, or not-for-profit sectors.

\section{ORCID iDs}

Hammoda Abu-Odah https://orcid.org/0000-0002-8874-2599

Nizar Said https://orcid.org/0000-0002-9373-610X

Satish C. Nair https://orcid.org/0000-0003-2095-1893

Matthew J Allsop https://orcid.org/0000-0002-7399-0194

David Currow https://orcid.org/0000-0003-1988-1250

Khamis Elessi https: // orcid. org/0000-0002-9313-9401 
Ali H. Alkhatib https://orcid.org/0000-0001-5290-9257

Yousuf ElMokhallalati https: // orcid. org/0000-0003-0047-1374

\section{Hosted file}

Table1.docx available at https://authorea.com/users/347405/articles/473113-optimizingtranslation-of-health-research-into-practice-a-systematic-review-of-reviews

\section{Hosted file}

Table 2.docx available at https://authorea.com/users/347405/articles/473113-optimizingtranslation-of-health-research-into-practice-a-systematic-review-of-reviews

\section{Hosted file}

Figure1.docx available at https://authorea.com/users/347405/articles/473113-optimizingtranslation-of-health-research-into-practice-a-systematic-review-of-reviews

\section{Hosted file}

Figure 2.docx available at https://authorea.com/users/347405/articles/473113-optimizingtranslation-of-health-research-into-practice-a-systematic-review-of-reviews 